\title{
The Life Style amongst Nomadic Tribes of Ivan Kalhor, Iran
}

\author{
Aida Mehrad \\ Faculty of Psychology \\ Universitat Autonoma de Barcelona \\ aida_238@yahoo.com \\ Spain \\ (Correspinding author) \\ Mahmoud Riyahi \\ Payame Noor University, Iran \\ azmon85@yahoo.com
}

\begin{abstract}
Cooperation is one of the main factors amongst nomadic tribes that caused of their unity and growth; also, it has been determined their life style. Furthermore, the goal of the current literature review was to explain the role of cooperation amongst people of tribes in Ivan Kalhor, Iran; also, illustrated the importance of this humanity factor in views of various famous scientists in the world that determined based on qualitative method. Lack of consideration to some factors such as cooperation in this kind of societies creates the different type of problems, and it can be harmful to nomadic tribes; additionally, it can decrease this population. In another word, the existence of cooperation as one of the principle human needs can advance and support activity and relation of people in tribes and conduct their activity in the correct way. Overall, this literature review that organized by qualitative method (observation and interview) considered to the vital role of cooperation at nomadic tribes in Iran especially in Ivan Kalhor area, and likewise, described its effectiveness on people relationships.
\end{abstract}

Keyword: Nomadic Tribes, Cooperation, Ivan Kalhor, Iran

\section{INTRODUCTION}

In the world population divided in various groups and each of them live based on their roles and cultures. All of these groups have one main purpose that has been originated from their human being. In actual fact, the individuals have various intentions for living and making better the life situation in different societies. One of the principle aims that can improve and also increase the level of individuals' life is cooperation that in each group has been defined differently. In addition, the cooperation has been changed in diverse years and appeared in dissimilar forms in varied population (Karimi dermani, 2010). One of the main population that focused on the cooperation as basic social factor in Iran is Nomadic tribes that have been altered widely in during the past; also, it has been defined differently. These changes in each group of them are approximately dissimilar, but as compared with city life are so less; additionally, they have preserved traditional aspects. These changes likewise in Nomad tribes of Iran were not considerable as compare with other country, because most of them have been selected modern life or modernity; in this regard, the type of their life style and relation has 
been well-defined in a different way. Furthermore, focusing on cooperation in each society or group is completely different and originated from population approach that living in individually society (Riyahi, 2015).

Generally, in the present study, the researchers just considered to the cooperation that performed in nomadic tribes in west of Iran. In truth, they wanted to reveal Iranian culture and relationships in nomadic tribes' area (Ivan Kalhor, Iran). Likewise, in the current study has been shown some real pictures in nomadic tribes of Iran for making clear their situation and life style. Picture 1 shows the lifestyle and condition of nomadic tribes in Ivan Kalhor, Iran.

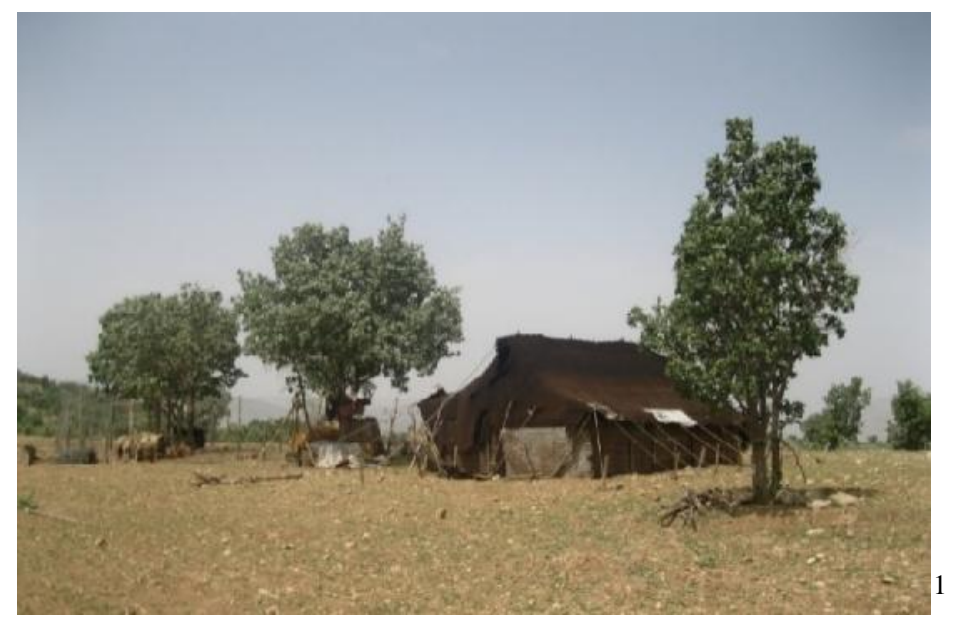

Picture 1. The lifestyle of Nomadic Tribes in Ivan Kalhor, Iran

\section{History of Nomadic Tribes}

In the early $19^{\text {th }}$ century, in Ivan Kalhor ${ }^{2}$, Iran, around 40 percentage of nomadic determined as "Emigrant Nomadic" that this amount has been decreasing in the $20^{\text {th }}$ century and appeared as 30 percentage of the population. Nomadic tribes have been included 8 groups in Iran. These groups involved Kurds, Lors, Persian, Turkish, Turkmen, Arabs, Baluch, and Brahui. Based on latest statistics, the number of nomadic tribes in west of Iran (Kurds) are around $7,000,000$. In fact, this amount included 7 percentage of total amount of population in this country. The rest of them changed their life style and have been selected city life and modernity. In reality, with the passage of time the individuals selected type of their life based on their interests and skills. The individuals that moved to cities preferred to developed their abilities in different ways and have relationship with various group of people. On the other hand, the people who stayed at tribes have been continued the pervious life style and followed the suggestions of elder people who lived in the tribe. But all of these people in each tribe did not have same condition, because the type of nomadic it was different (Farhadi, 1994).

\footnotetext{
${ }^{1}$ All the pictures have been taken by the researcher Mahmoud Riyahi

${ }^{2}$ Ivan Kalhor is one of the principle Nomadic areas in west of Iran
} 


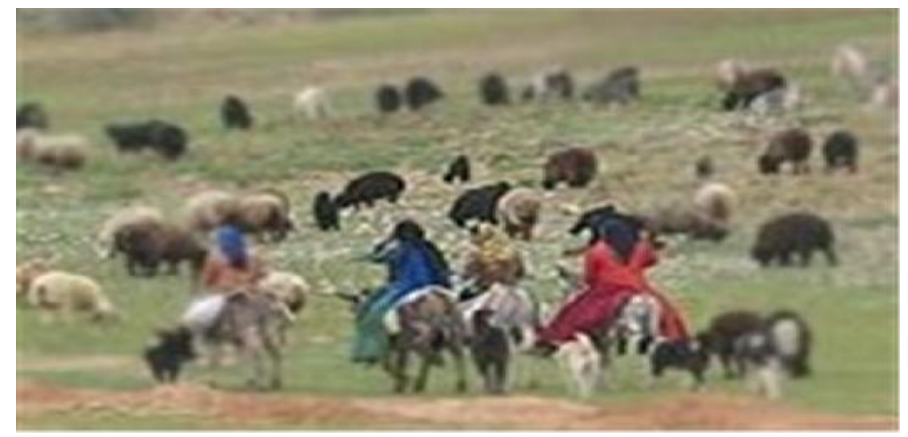

Picture 2. Nomadic tribes' life style

\section{Nomadic Groups}

The nomadic divided into three groups. These groups included: 1) Immigrant (this group of nomadic population determined as natural lifestyle in Iran).

2) Settled population (this group of nomadic likewise has been set as population that settled in nomadic villages).

And finally, 3) Semi-settled (this group of nomadic spending 6 months in village and 6 months in hood) (Riyahi, 2015). In continue, Picture 3 illustrates this area in the map of Iran (Ivan Kalhor, Iran).

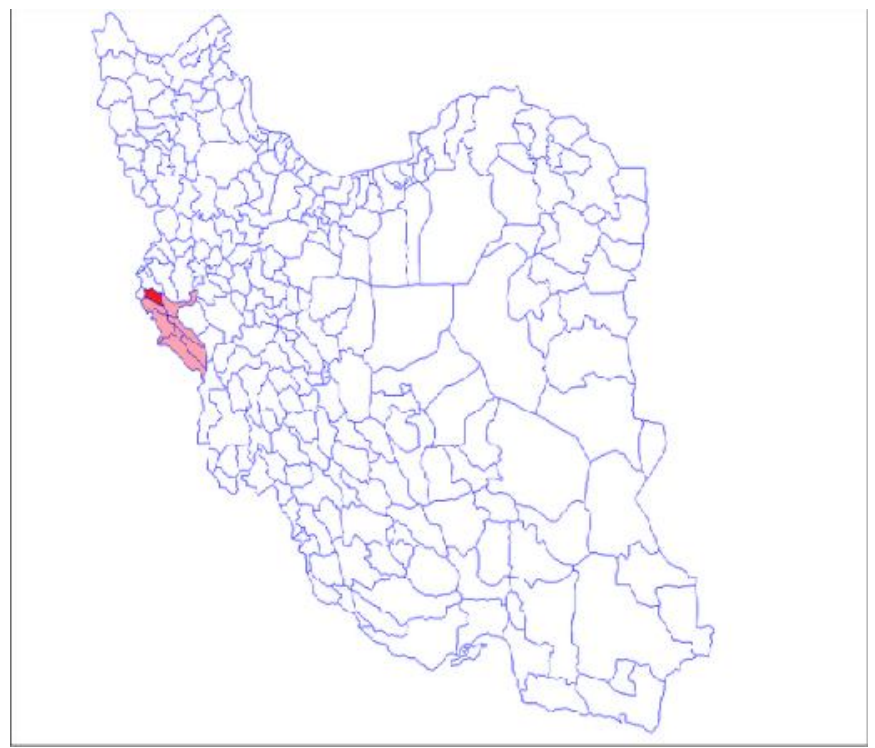

Picture 3: Ivan Kalhor, Iran

\section{Culture of Nomadic Tribes and Role of Cooperation}


The culture of nomadic tribes amongst people in Ivan Kalhor area has been determined based on previous traditions and speeches of elders that have been transferred one by one. These traditions have involved animal husbandry, agricultural, weddings, migration, mourning, etc. that people based on them spending their life and have cooperation together. The cooperation defined as working together for specific aim and satisfying human requirements. Additionally, this behavior well-defined as the achievement or process of working together to the similar end. Defiantly, the cooperation amongst nomadic assumed as the main human factor that has considerable importance. This cooperation is a mutation in the way of unifying in front of competition. In actual fact, when the cooperation presences in any population the completion is meaningless (Karimi Dermani, 2010).

The cooperation amongst people of nomadic tribe explained that each of them has noticeable responsibility for making better condition; and never focused on personal benefits (Bahonar, 2009). In reality, the cooperation explained intention for obtaining the goal that organized via specific individuals such as elders or leaders in the tribe (Dehkhoda, 1998). Picture 4 likewise displays the cooperation among nomadic people.

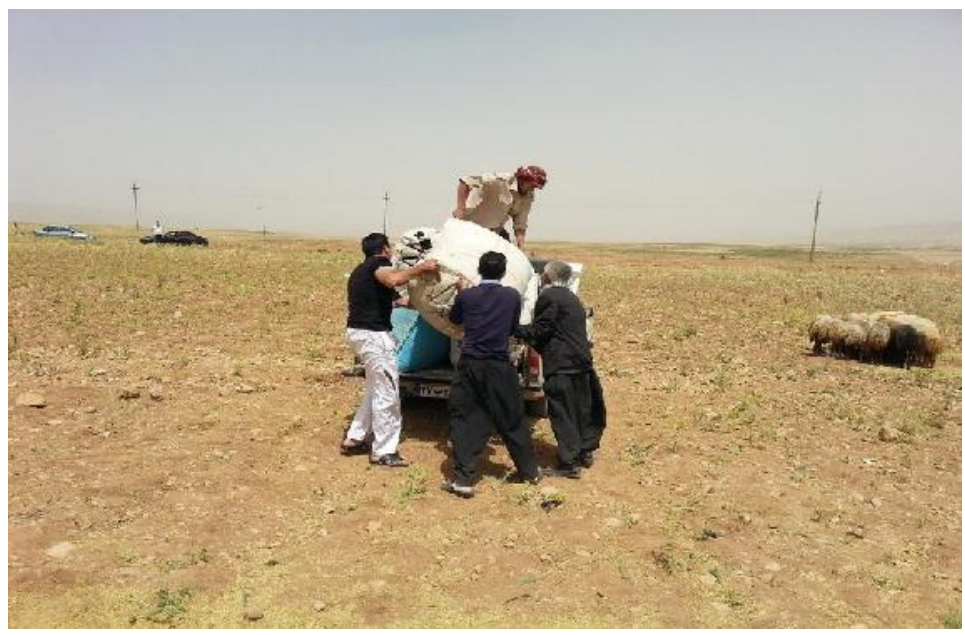

Picture 4: The Cooperation amongst Nomadic People

\section{The Views of Scientists about Cooperation}

The importance of cooperation has been emphasized by various scientists in the world. One of the brilliant sociologists that has categorized cooperation is Ibn Khaldun (1332-1406). He divided cooperation in two group: 1) the cooperation with the division of labor, and 2) the cooperation without the division of labor. In nomadic tribe society, the cooperation appears as a voluntary basis. In real, the cooperation organized based on security, mental and social needs satisfaction, and advancement that people show it in their social life (Ansary, 1992). In this regard, some scientists such as Aflaton, Aristotle, Farabi, Avicenna or Ibn-Sina, Khawaja Muhammad ibn Muhammad ibn Hasan Tusi, and August Kont have been insisted on necessary and imperative of cooperation among people in various societies. They explained that the cooperation can be useful for society and reduces difficulties in the environment; also, 
it can develop people's ability. These scientists believed that the cooperation is caused of advancement in every civilization and increases the amount of activities and motivations of people. The scientists described that presence of cooperation among people can be effective on their relationship and also their life.

In Iran also the presence of cooperation has a long history and has continued to this day. In reality, the cooperation as standard social factor has been reserved in all populations in the world. As a result of the passage of time, the people in all the world have been founded that human relationships can be operative on individually life; additionally, they assumed as positive motivators in lifecycle. In this regard, considering to the cooperation as main and affirmative behavior is very imperative. And it should be perform and emphasize in all cultures (Froghy, 1990). In the following, Figure 1 shows the relation between cooperation and people's ability.

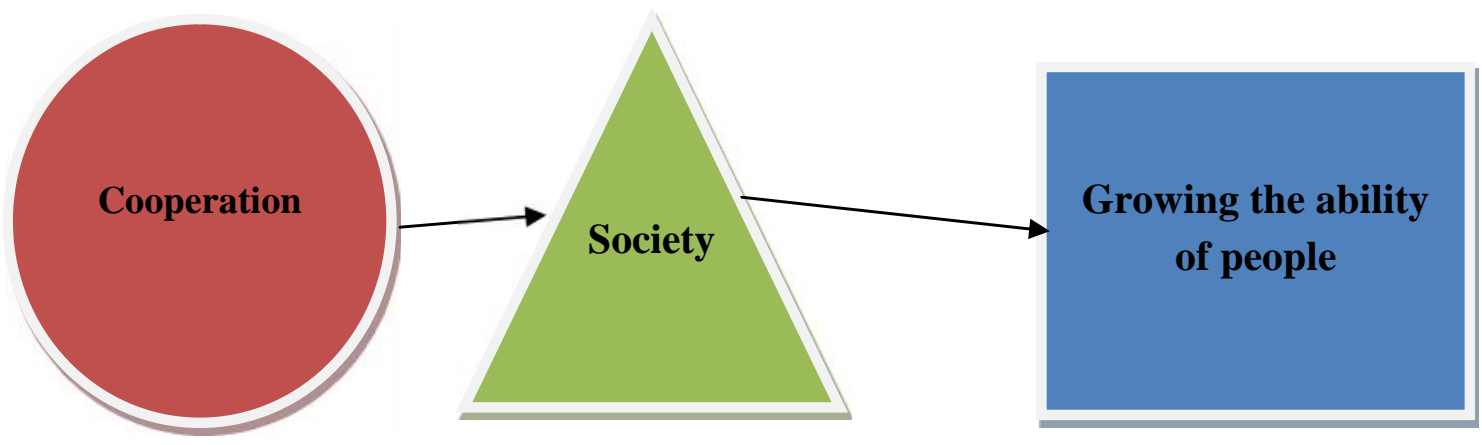

Figure 1. Role of cooperation on people's ability in society

\section{CONCLUSION}

Cooperation, as mentioned before, is so essential in the nomadic tribes; moreover, lack of attention to this factor and also a low level of it appeared different difficulties amongst the people in the tribe. Also, it reduces their relation and activity in daily life. These problems are serious threats to the tribe and their members. In fact, lack of cooperation appear hard situations in the tribe, and the people cannot do their activities as usual because most of them needed cooperation. Furthermore, for saving cooperation and conduct it in an accurate way, the elders in each tribe should determine the responsibility of each person and advise them correctly. Additionally, they should distinguish the needs of people based on their life situation. Likewise, the people should do their responsibility and have a unit aim to obtain it immediately and preserve their relation based on tribe policies. In the main, the current 
literature review described importance of cooperation amongst people of the nomadic tribe that can improve and increase the levels of their relations and activities.

\section{REFERENCE}

Ansary, H. (1992). Principles of cooperation. Tehran: Payame Noor University.

Bahonar, N. (2009). Media and religion ( $1^{\text {th }}$ ed.). Tehran: Research and Evaluation Center IRIB.

Dehkhoda, A. A. (1998). Dehkhoda dictionary (6 ${ }^{\text {th }}$ ed.). Tehran: Tehran University.

Farhadi, M. (1994). Culture of corporation in Iran: Introduction to anthropology and sociology of cooperation. Tehran: University Press

Karimi dermani, H. R. (2010). Fields and methods of cooperation in Iran (1 $1^{\text {th }}$ ed.). Tehran: KandoKav

Riyahi,M. (2015). The Ways of cooperation in nomadic. Tehran: AzmonyarPooya.

Froghy, M. A. (1990). The passage of wisdom in Europe ( $1^{\text {th }}$ ed.). Tehran: Safi Ali Shah.

\section{Interview Part:}

Yasemy, Y. (2015). One of the tribesman in Ivan Kalhor

Esmaieli, N., \& Khani, A. R. (2015). The teachers of Ivan

Yasemy, K., \& Riyahi, GH. R. (2015). The elders in Ivan 